\title{
SISTEMAS DE GERENCIAMENTO DE BANCO DE DADOS EM TEMPO- REAL NA ÁREA HOSPITALAR: UM ESTUDO DE CASO PARA UNIDADE DE TERAPIA INTENSIVA
}

\section{Yáskara Ygara Menescal Pinto Fernandes}

Mestre pelo Programa de Pós-Graduação em Engenharia Elétrica, da Universidade Federal de Campina Grande, 2005. Atualmente é Professora da Universidade Federal Rural do

Semiárido. Email: yaskaramenescal@ufersa.edu.br

Pedro Fernandes Ribeiro Neto

Doutor em Engenharia Elétrica pela Universidade Federal de Campina Grande - UFCG (2006). Mestrado em Engenharia Elétrica pela Universidade Federal de Campina Grande UFCG (2001). Professor da Universidade do Estado do Rio Grande do Norte, Adjunto IV, no Departamento de Informática. Professor Permanente do Programa de Pós-Graduação em Ciência da Computação - Associação Ampla UERN/UFERSA.

Email: pedro.fernandes2503@gmail.com

\section{Antonio Higor Freire de Morais}

Professor de Sistemas de Informação do Instituto Federal de Educação, Ciência e Tecnologia do Rio Grande do Norte (IFRN), Doutorando e Mestre em Engenharia Elétrica e de Computação pela Universidade Federal do Rio Grande do Norte (UFRN).

E-mail: higor.morais@ifrn.edu.br

\section{Ricardo Alexandro de Medeiros Valentim}

Doutor em Engenharia Elétrica e de Computação, Coordenador do Laboratório de Inovação Tecnológica em Saúde do Hospital Universitário Onofre Lopes, Professor do Departamento de Engenharia Biomédica da Universidade Federal do Rio Grande do Norte.

E-mail: ricardo.valentim@ufrnet.br

\section{RESUMO}

A automação hospitalar consiste em uma área que está em constante crescimento. Os processos envolvidos na mesma estão sendo informatizados e automatizados cada vez mais com novas tecnologias tanto de software como de hardware. Fato este, que implica no aumento do número de informações e complexidade no desenvolvimento de sistemas e gerenciamento de dados. Nesse sentido, várias pesquisas têm sido desenvolvidas e geralmente tem abordado vários dos problemas que são pertinentes aos processos que podem ser automatizados, tais como: segurança, comunicação, confiabilidade e desempenho das aplicações, dispositivos biomédicos, usabilidade dos sistemas, consistência lógica e temporal dos dados transações, entre outros. Considerando tais problemas, os sistemas de gerenciamento de banco de dados em tempo-real (SGBD-TR) apresentam características necessárias para garantir a validade lógica e temporal dos dados e transações, além de serem projetados para gerenciar grande volume de dados. Os SGBDTR podem ser aplicados em: automação hospitalar, aviação, redes de sensores, entre outras. Essas aplicações possuem como característica o fato de, precisam executar em ambientes abertos e imprevisíveis por apresentar grande distribuição geográfica, alta heterogeneidade, inexistência de controle global, falhas parciais e falta de segurança. Portanto, considerando a complexidade dessas aplicações, torna-se fundamental realizar um estudo de caso em unidade de terapia intensiva (UTI), onde buscamos automatizar dados e transações em tempo-real com restrições temporais, considerando a consistência lógica e temporal dos mesmos. Dessa forma, serão utilizadas técnicas de qualidade de 
serviço para definir os níveis de corretude e de desempenho que devem ser satisfeitos pelas aplicações. Através do estudo de caso é possível realizar análises e simulações, buscando orientar o processo de tomada de decisão e propor soluções para a melhoria.

PALAVRAS-CHAVE: Automação hospitalar, Sistemas de banco de dados em tempo-real, qualidade de serviço.

\title{
REAL-TIME DATABASES SYSTEMS IN HOSPITAL AREA: A CASE STUDY FOR INTENSIVE CARE UNITS
}

\begin{abstract}
Hospital Automation is an area that is constanlty growing. The emergency of new technologies and hardware is transforming the processes more efficient. This fact, which implies increasing the number and complexity of information systems development and data management. To this end, several studies have been developed and is usually addressed most of the problems involved in processes with automation potential, such as: security, communication, reliability and performance of applications, biomedical devices, systems usability, logical and temporal consistency, among others. Considering these problems, the database in real-time (RTDB) have the necessary characteristics to ensure logical and temporal validity of the data and transactions, and are designed to manage large volumes of data. The RTDB can be applied as: hospital automation, aviation, sensors network, and so on. These applications have the characteristic fact, need to run in open and unpredictable by presenting large geographic distribution, high heterogeneity, lack of global control, partial failures and lack of safety. Therefore, considering the complexity of these applications, it is essential to conduct a case study in intensive care unit (ICU), where we seek to automate data and real-time transactions with timing constraints, considering the logical and temporal consistency of the same. Thus, techniques will be used to define quality of service levels and correctness of performance that must be met by applications. Through the case study it is possible to perform analysis and simulations to guide in the process of decision making and propose solutions for improvement.
\end{abstract}

KEYWORDS: Automation hospital database in real-time, quality service.

\section{SISTEMAS DE GERENCIAMENTO DE BANCO DE DADOS EM TEMPO- REAL NA ÁREA HOSPITALAR: UM ESTUDO DE CASO PARA UNIDADE DE TERAPIA INTENSIVA}

\section{INTRODUÇÃO}

Os hospitais vêm ao longo do tempo informatizando os seus processos. Para tanto, fazem uso de sistemas de informação que automatizam algumas tarefas pertinentes ao ambiente hospitalar. Muitos destes sistemas são, em sua grande maioria, dirigidos à gestão e, portanto buscam a redução dos custos e a otimização dos processos administrativos. Normalmente os hospitais fazem uso de alguns dos seguintes sistemas: prontuário eletrônico; marcação de consulta; controle de farmácia; internamento; laboratoriais; entre outros (Araújo, 2010). 
Diante desse contexto, a área hospitalar passou também a incorporar conceitos aplicados na automação (Brooks e Brooks, 1998), buscando garantir o aprimoramento na execução de operações referentes a procedimentos médicos. Um forte exemplo desta tendência é o desenvolvimento de pesquisas voltadas ao monitoramento de pacientes, as quais através da aquisição de dados realizam operações de controle aplicadas ao monitoramento dos sinais vitais (Pizarro, 2003). No entanto, esses sistemas processam grandes volumes de dados e transações, onde os prazos temporais impostos aos mesmos não podem ser perdidos sob a pena de gerar algum dano ao paciente.

O monitoramento de pacientes internados em unidade de terapia intensiva (UTI) é considerado uma tarefa crítica, pois envolvem dados e transações que estão relacionados com saúde do paciente, indicando o quadro clínico do mesmo (Leite et al., 2011). Então, é necessário o uso de mecanismos de gerenciamento de dados e transações em tempo-real para que as mesmas sejam processadas de forma consistente em limites determinados de tempo (não necessariamente significa que o tempo deve ser rápido). Isto significa que um determinado evento no banco de dados deve ser cumprido em um limite máximo de tempo, cujo descumprimento pode trazer prejuízos diversos ao sistema.

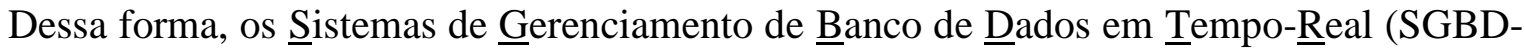
TR) apresentam como característica o tratamento de dados e transações que possuem restrições de tempo não negociáveis, tais como aplicações em aviões e automóveis, monitoramento de pacientes, onde nessas aplicações os prazos temporais impostos aos dados e as transações não podem ser perdidos sob a pena de gerar alguma catástrofe (Lindstrom, 2008).

Nas últimas duas décadas, pesquisas sobre SGBD-TR de modo a determinar métodos, técnicas e soluções para tratar com a complexidade imposta pela necessidade de garantir a integridade dos dados e satisfazer as restrições de tempo das transações têm sido realizadas. Assim, novas abordagens têm sido introduzidas no escopo de SGBD-TR com o objetivo de tratar com os diversos problemas decorrentes dos requisitos impostos, dentre os quais destacamos: Métodos para modelagem conceitual, Protocolos de controle de concorrência, algoritmos de escalonamento e linguagem de consulta (Idoudi, et al.,2009; Lindstrom, 2008; Fernandes, 2005; Ribeiro Neto, 2006).

Algumas pesquisas em relação ao monitoramento de pacientes em tempo-real têm sido desenvolvidas (Murakami, et al., 2006; Varshney, 2006 ). No trabalho de (Pizarro, 2003), foi desenvolvido um monitor de multi-parâmetros remoto, o qual possibilita o monitoramento em tempo-real de sinais vitais de pacientes através de uma rede IP, como a Internet. No entanto, em tais pesquisas não foram considerados o tratamento de dados e transações em tempo-real no monitoramento de pacientes, ou seja, é importante observar que, os dados gravados em um SGBD-TR devem ter valor idêntico ao que está monitorando no ambiente real para que uma tomada de decisão seja realizada com maior precisão.

Dessa forma, o objetivo do artigo é apresentar um estudo de caso em monitoramento de pacientes na Unidade de Terapia Intensiva (UTI), utilizando técnicas de SGBD-TR para que os dados e transações executem dentro das restrições de tempo garantindo a consistência lógica e temporal dos mesmos. 
A utilização de mecanismos de qualidade de serviço (QoS) para o gerenciamento de dados e transações em tempo-real é necessária para especificar a qualidade requerida em termos de quão impreciso pode ser o dado, para ainda ser considerado válido e qual o limite de perdas de prazo das transações para o desempenho do sistema ainda ser aceitável.

\section{MONITORAMENTO DE PACIENTES EM TEMPO-REAL}

Nos últimos anos, tem-se presenciado as mudanças significativas trazidas pelo desenvolvimento da tecnologia da informação. Estas mudanças foram importantes para a modernização de muitas áreas do mundo dinâmico e moderno. A biomedicina não foi exceção, onde a importância da tecnologia da informação nesta área tem sido amplamente reconhecida e suas aplicações tem se expandido para além do limite da automatização dos serviços prestados e processos no ambiente hospitalar, levando à descoberta de conhecimento nas ciências da vida e da medicina.

Entretanto, as novas descobertas na área médica têm salvado muitas vidas através de novos parâmetros, processos, técnicas, abordagens, entre outros. E a integração das ciências, medicina e tecnologia estão se tornando um importante motivo para o aprimoramento do desenvolvimento da tecnologia da informação. Muitas áreas emergentes foram recentemente desenvolvidas, incluindo a informática da saúde, bioinformática, engenharia biomédica, informática de imagem (ou até mesmo de informática de imagens médicas), biometria médica, sistemas de informação para automação hospitalar, dentre outros (Feng, 2007).

Ultimamente algumas pesquisas têm desenvolvido trabalhos relevantes aplicados à automação hospitalar. Dentre dos quais podemos destacar (Marani, 2012):

- Sistemas para o monitoramento de pacientes;

- No uso de tecnologias de informação para melhorar o nível de usabilidade dos sistemas hospitalares;

- Na definição de arquiteturas de redes para a transferência de sinais biomédicos e de dados;

- No desenvolvimento de biodispositivos e biosensores;

- Na especificação de protocolos aplicados a uma área médica específica.

Com relação ao monitoramento de pacientes, os trabalhos normalmente são dirigidos à automatização deste processo através de sistemas que utilizam biodispositivos ou biosensores para fazer a aquisição dos sinais vitais dos pacientes. $\mathrm{O}$ objetivo é permitir que os membros da equipe médica tenham acesso a esses sinais e possam então realizar os procedimentos médicos com maior segurança e precisão.

No contexto de sistemas de monitoramento, (Murakami et al.;2006) desenvolveu o vMonGluco que implementa o monitoramento em tempo-real dos níveis de glicose dos pacientes. Este sistema foi desenvolvido sobre dispositivos móveis, sendo utilizado em UTI. Esse trabalho apresenta bons resultados, pois permite que pacientes com altas taxas de glicose possam ser monitorados de forma automatizada e com uma frequência maior e mais precisa, melhorando a qualidade do atendimento do paciente e também possibilitando o escalonamento mais eficiente da equipe médica, uma vez que um processo que demandava tempo e recursos humanos foi automatizado. Apesar de propor e demonstrar a ferramenta utilizada para medir os níveis de glicose dos pacientes, Murakami não aborda em seu trabalho um mecanismo que garanta que as medições dos níveis de glicose do paciente serão de fato mostradas nos períodos desejados. Portando, o vMonGluco não 
oferece garantias de que as informações serão de fato entregues, ou que serão entregues nos prazos estabelecidos, ou seja, dentro das restrições de tempo.

Seguindo também a linha de monitoramento de pacientes, (Varshney, 2006) apresenta alguns requisitos, e propõe um modelo orientado a redes wireless para processo de monitoramento de pacientes. Um aspecto bastante positivo deste trabalho é a utilização do padrão IEEE 802.11, que é aberto e tem se tornado bastante popular, com fácil acesso e baixo custo.

Todavia, (Varshney, 2006) não considera no trabalho os problemas provenientes dos ambientes hospitalares para este tipo de tecnologia, tais como: ruído, interferência ou perda de sinal. Os sinais que as redes wireless propagam estão mais suscetíveis a ruído, visto que o meio físico é o ar, e a interferência ou a perda de sinal podem ser ocasionadas por diversos motivos, por exemplo, as salas de Raio- $\mathrm{X}$ e de Tomografia que são revestidas com chumbo e portanto podem ser um obstáculo isolante do sinal das redes wireless.

A interferência pode ser representada pelo impacto que este tipo de transmissão pode ocasionar em um dispositivo médico, ou também sofrer de outros dispositivos hospitalares. Os sistemas de monitoramento sem fios que são utilizados por muitos hospitais podem ser potencialmente mortais criando falhas de dispositivos médicos, como por exemplo, respiradores, máquinas de diálise e marca-passo externo (Neumann, 2008).

O monitoramento de pacientes, apesar de ser um processo lento, quando comparado aos da indústria, demandam, no entanto uma observação contínua e com uma frequência determinada pelo tipo de monitoramento e gravidade na qual o paciente se encontra, (Baura, 2004) e (Brooks e Brooks, 1998). Esse aspecto é um requisito que exige dos sistemas de monitoramento garantias de disponibilidade e corretude temporal. Nesse contexto, os trabalhos citados apresentam soluções aplicadas ao monitoramento de pacientes nos processos da automação hospitalar. Entretanto, as pesquisas apontadas não apresentam em suas propostas mecanismos de qualidade de serviço que garantam que os sinais adquiridos através do processo de monitoramento de pacientes, serão de fato entregues dentro das restrições de tempo que um determinado dado e transações precisam executar.

Dessa forma, o SGBD-TR apresentam características para justificar que as aplicações de monitoramento de pacientes por terem tais requisições devem ser executadas sob mecanismos de troca de dados que garantam uma execução eficiente do processo, visto que um mau funcionamento dos sistemas de monitoramento pode gerar prejuízos à vida humana.

\section{SISTEMAS DE GERENCIAMENTO DE BANCO DE DADOS EM TEMPO- REAL (SGBD-TR)}

Nos últimos anos, várias pesquisas têm sido realizadas na área de SGBD-TR (J. Hansson e S. H. Son, 2001; Wang et al., 2012; Shanker et al., 2008; Fernandes, 2005), uma vez que esses sistemas tratam com aplicações que envolvem o gerenciamento de grandes quantidades de dados, além de permitir tratar com dados e transações com restrições em tempo-real. Os SGBD-TR surgiram com uma publicação especial, registrado em $A C M$ SIGMOD em março 1988. 
Atualmente, muitas aplicações requerem o uso de tais sistemas, dentre elas: os sistemas de recuperação da informação, os sistemas do reserva de passagem aérea, sistemas bancários, sistemas de controle de avião e de nave espacial, robótica, a automação de fábrica e de hospital, dentre outros (Squadrito, 1996).

A importância funcional dos SGBD-TR se dá pelo fato destes suportarem as características de um sistema de banco de dados (SGBD), ou seja, de processar transações e garantir a consistência lógica dos dados e transações, como também as características de um STR, de satisfazer às restrições de tempo impostas as tarefas (Shanke et al., 2008).

As principais diferenças entre um SGBD tradicional e um SGBD-TR são: o gerenciamento de dados temporalmente consistentes, ou seja, dados que são válidos apenas por um período de tempo específico e o gerenciamento de transações de forma que elas executem dentro de um prazo definido a priori. As restrições temporais podem ser originadas da necessidade de rastrear continuamente um ambiente ou da necessidade de fazer com que os dados do sistema controlado estejam disponíveis para suas atividades de tomada de decisão (Ramamritham, 2004).

Os SGBD-TR podem ser utilizados com sucesso em aplicações com restrições de tempo que precisam armazenar, modificar e recuperar grandes volumes de dados compartilhados. Dentre estas aplicações estão comércio eletrônico, bolsa de valores on-line e várias aplicações de redes de sensores (J. Hansson e S. H. Son, 2001). Por exemplo, considere um ambiente monitorado por sensores, onde esses adquirem mudanças de estado do ambiente. Os valores adquiridos possuem validade temporal e as transações possuem prazos.

A corretude dos dados em um SGBD-TR é garantida pela consistência lógica e temporal. Contudo, nem todos os dados possuem validade temporal, sendo possível classificá-los em dados sem restrições de tempo-real, ou simplesmente dados atemporais e dados com restrições de tempo-real ou dados temporais (Jha et al., 2008). A corretude dos dados atemporais é garantida somente pela sua consistência lógica, uma vez que não possuem restrições temporais. Os dados temporais surgem da necessidade de refletir o estado do ambiente que está sendo controlado no banco de dados.

As transações nos SGBD-TR podem ser categorizadas quanto às restrições de tempo-real, quanto aos padrões de chegada e quanto ao tipo de acesso aos dados. Esta classificação é muito importante para o gerenciamento dos dados e das transações, uma vez que a prioridade, periodicidade e conflitos podem ser definidos (Lindstrom, 2008).

- Restrições de tempo-real

As restrições de tempo-real das transações podem ser estritas, suaves e firmes. Um prazo restrito é atribuído às transações que devem atender seus prazos, caso contrário, podem provocar consequências catastróficas. Uma transação com prazo suave pode completar após o seu prazo. No entanto, não cumprir prazos suaves pode comprometer o desempenho do sistema. Já as transações com prazos firmes serão abortadas se as restrições temporais definidas não forem atendidas.

- Padrões de chegada das transações 
Os padrões de chegada das transações se referem ao intervalo de tempo em que elas podem iniciar suas execuções. As transações podem ter padrões de chegada periódicos onde existe um intervalo de tempo regular entre duas execuções consecutivas da mesma transação. As transações também podem possuir padrões de chegada aperiódicos, onde não existe um intervalo de tempo regular entre suas execuções. Por fim, as transações podem ser esporádicas que são iniciadas em instantes de tempo aleatórios. No entanto, existe um intervalo mínimo entre duas execuções consecutivas da mesma transação.

- Tipo de acesso aos dados

Quanto ao tipo de acesso aos dados, as transações podem ser classificadas como: transações de escrita (ou sensores) e transações de leitura. As transações de escrita obtêm o estado do ambiente e escrevem os dados no banco de dados. Essas são tipicamente periódicas. As transações de leitura leem dados do banco de dados, podendo ser periódicas ou aperiódicas.

\section{ESTUDO DE CASO EM UNIDADES DE TERAPIA INTENSIVA (UTI)}

É crescente a necessidade de sistemas que façam um completo e transparente monitoramento das informações nos processos envolvidos, para que não dificulte e atrase a execução das atividades relacionadas (Carvalho, 2005). Qualquer ganho no tempo de execução dessas atividades é importante, principalmente em sistemas que envolvam grande volume de informações, ou sistemas críticos. Novas tecnologias surgem a cada dia para dar suporte a esses tipos de processamento, mas geralmente são limitadas áreas a serem utilizadas em sistemas heterogêneos, como é o caso do hospital.

O ambiente hospitalar apresenta um grande volume de dados, os quais, na maioria das vezes, são considerados críticos, pois estão relacionados com a saúde de um paciente. Esses dados variam constantemente, podendo apresentar anormalidades, as quais devem ser detectadas em curto espaço de tempo e informadas o mais rápido possível ao profissional responsável pelo paciente.

É o caso dos sinais vitais de um paciente que esteja internado em UTI, pois quando ultrapassam determinados limiares, representa alguma anormalidade, e a vida do paciente pode estar em risco. O tempo para que um alerta seja gerado e chegue até o profissional também deve acontecer em tempo aceitável, para que se possa tomar uma decisão rápida e adequada em relação aos procedimentos preconizados nos protocolos de atendimento médico.

Os SGBD-TR podem ser aplicados em monitoramento de pacientes, a fim de garantir que dados atuais coletados durante um alerta gerado, sejam utilizados por transações válidas temporalmente.

Na Figura 1 é mostrado um cenário do sistema, considerando o domínio de aplicação de monitoramento de pacientes em UTI, que consiste de um sistema controlador e um sistema controlado.

O sistema controlador é composto pelo computador servidor, e pelas interfaces humanas (Profissional da Saúde), enquanto que o sistema controlado representa o ambiente a ser controlado, podendo ser uma fábrica, um armazém, hospital, poços de petróleo (Lindstrom, 
2003). Nesta arquitetura, os dados com suas restrições temporais, bem como as transações com suas restrições e categorizações são analisados.
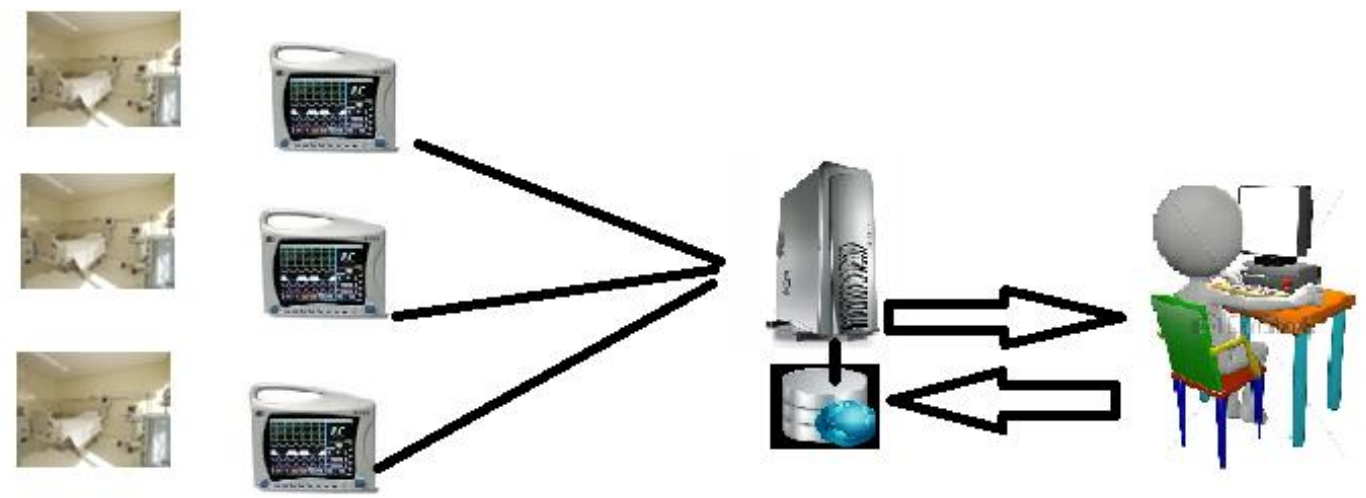

Figura 1 - Cenário do Sistema em UTI

O sistema controlador é composto pelo computador servidor, e pelas interfaces humanas (Profissional da Saúde), enquanto que o sistema controlado representa o ambiente a ser controlado, podendo ser uma fábrica, um armazém, hospital, poços de petróleo (Lindstrom, 2003). Nesta arquitetura, os dados com suas restrições temporais, bem como as transações com suas restrições e categorizações são analisados.

O módulo SGBD-TR está de acordo com o descrito em (Brayner, 1999) e (Ribeiro Neto, 2006) composto pelos seguintes componentes, como apresentado na Figura 2: gerenciador de transações, escalonador, gerenciador de recuperação e gerenciador de cache. As transações são recebidas e encaminhadas para o escalonador através do gerenciador de transações. No escalonador é feito o controle da execução concorrente das transações. $\mathrm{O}$ gerenciador de cache tem como finalidade manter o cache, movendo dados da unidade de armazenamento volátil para a estável e; o gerenciador de recuperação é responsável por garantir que o banco de dados mantenha todos os efeitos de uma transação confirmada e nenhum dos efeitos de uma transação abortada.

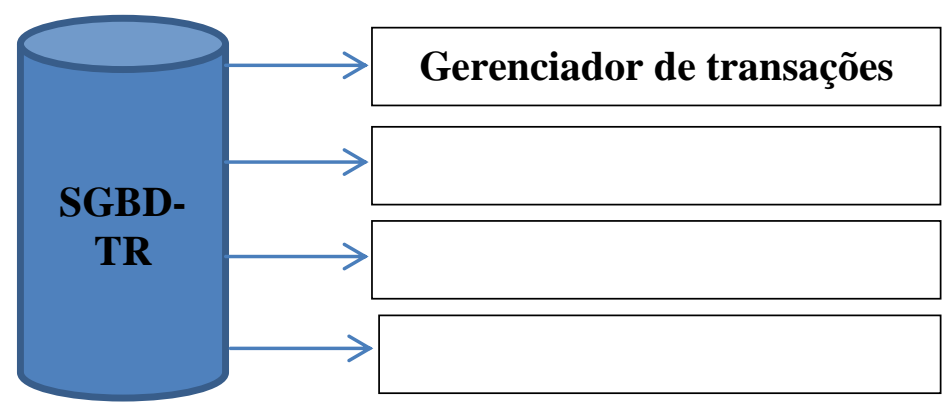

Figura 2 - Módulo SGBD-TR

O estudo de caso consiste em um sistema para alertar aos profissionais da saúde (PS), quando um paciente se encontra em um estado crítico (Codigo_vermelho), e 
"imediatamente", de acordo com as restrições de tempo fornecê-los as estatísticas completas e atuais sobre o estado do paciente (como sangue e análise da urina ou quaisquer outros testes que estão rotineiramente feito sobre o paciente, e as estatísticas vitais que estão a ser constantemente monitorizados, por exemplo, pressão sanguínea e temperatura). O banco de dados é amplamente distribuído.

O sistema apresenta três unidades básicas. A unidade de processamento de dados contém as informações habituais dos pacientes:

- O Paciente (Codigo_Paciente, nome, endereço, parentesco, NumTelefone, ....).

O laboratório de teste contém resultados de testes realizados em pacientes:

- LAB (Código_Paciente, Data_teste, tipo_de_teste, resultados).

A unidade de terapia intensiva contém um histórico de estatísticas vitais em pacientes criticamente enfermos:

- UTI (Codigo_Paciente, NumCama, temperatura, pressão_arterial, pulso, ...).

Um relatório atual (Codigo_vermelho) a respeito dos pacientes que ocupam os leitos da UTI deve ser disponibilizado para o profissional de saúde:

- Codigo_vermelho (codigo_paciente, nome, NumCama, parentesco, Data_teste, tipo_de_teste, resultados, temperatura, pressão_arterial, pulso).

Cada paciente é ligado a monitores dedicados de medições, como apresentado na Figura 1, que detectam as condições críticas (tal como um rápido aumento na pressão sanguínea). Quando uma condição crítica ocorre, um flag de alerta é ativado e o número da cama do paciente é enviado para o computador central com cuidados intensivos.

Note que, em determinado momento, qualquer quantidade de pacientes que ocupam leitos na UTI pode ter ativado uma alerta. Isso pode ser visto como um conjunto de relações $\left(\right.$ ALERTA $_{1}$, ALERTA $\left._{2}\right)$ onde ALERTA AL $_{\mathrm{i}}$, contém todos os números de cama que ativaram um alerta desde do tempo que ALERTA $_{i-1}$ foi ativado.

Dessa forma, considere uma situação de acordo com a Figura 3, onde o PS realiza uma consulta no paciente da cama 1, que se encontra em um estado crítico, para verificar "o mais recente" resultado do dado da pressão sanguínea, ou requisitar um histórico limitado dos resultados de cada exame, para obter uma visão geral de como o paciente está reagindo.

No entanto, neste mesmo instante de tempo a cama 1 e 2 simultaneamente ativam o alerta com os dados para atualizar a pressão sanguínea no banco de dados, com os dados recentes do paciente, como visto na Figura 3. Portanto, nesta situação, o PS não pode esperar uma quantidade excessiva de tempo para obter resposta completa. Uma consulta com resultado impreciso pode produzir informação suficiente para determinar que ação imediata deva ser tomada. 
Assim, de acordo essa situação existe um problema clássico de execução concorrente de transações conflitantes (uma transação de leitura e uma transação de escrita), tentando acessar o mesmo item de dado. Nessa circunstância uma forma de negociação deve ser realizada através de uma Função de Negociação (FN), onde a mesma permite a negociação entre consistência lógica e temporal dos dados e transações de acordo com as necessidades da aplicação, além de controlar o grau de imprecisão gerado no banco de dados quando a consistência lógica ou temporal for sacrificada. Como por exemplo, se a consistência lógica for escolhida, existe a possibilidade que o item de dado torne-se temporalmente inválido, ou que uma transação viole sua restrição temporal. Se por outro lado, consistência temporal for escolhida, a consistência lógica do dado ou da transação pode ser comprometida.

Portanto, a FN avalia os parâmetros temporais e parâmetros lógicos das operações. Nessa etapa verifica-se se os dados utilizados ainda são válidos temporalmente e se a imprecisão está dentro dos limites de imprecisão especificados. Se o dado é válido temporalmente e estão dentro dos limites de imprecisão, ou seja, então as duas operações serão executadas concorrentemente e a imprecisão é introduzida no banco de dados.
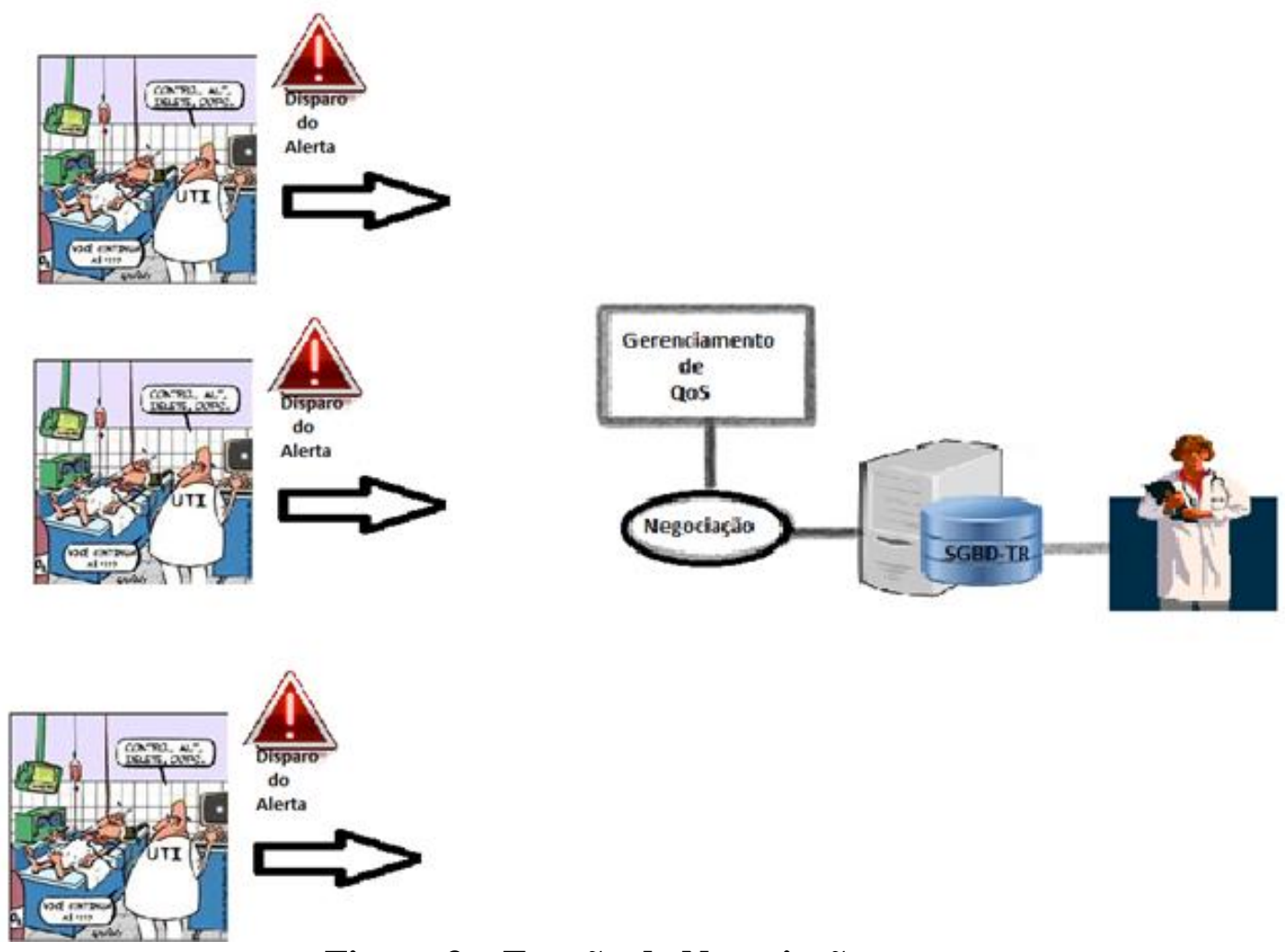

\section{Figura 3 - Função de Negociação}

Dessa forma, o PS consegue realizar a consulta, embora o dado apresente imprecisão que esteja dentro do limite especificado, e assim tomar uma decisão que não seja catastrófica.

No entanto, antes das transações completarem a sua execução, o gerenciamento de QoS é executado para verificar se a qualidade requerida pelo sistema está sendo atendida. As funções de QoS são utilizadas durante a execução das transações nos SGBD-TR, dentre as quais podemos citar (Amirijoo et al., 2006): função de especificação, função de mapeamento, função de negociação, função de monitoração e função de re-negociação. 
As transações de leitura e de escrita possuem os parâmetros temporais que são especificados através da função de especificação, onde através desta função são identificados os parâmetros de tempo e os parâmetros lógicos necessários para garantir o perfeito funcionamento do sistema. Uma vez identificados, alguns destes parâmetros são especificados a priori e outros em tempo de execução de acordo com a situação atual do sistema.

Dentre os parâmetros especificados inicialmente tem-se a periodicidade, o prazo e o tempo de liberação de uma transação. Em relação aos valores especificados em tempo execução está a imprecisão admitida no item de dado. Uma vez especificados, estes parâmetros serão mapeados (durante toda a execução da transação através da função mapeamento).

Quando ocorrer execução concorrente de transações conflitantes, uma negociação deve ser realizada onde uma função de negociação será invocada para fazer a análise, como foi apresentado anteriormente.

É necessário verificar se a qualidade requerida está sendo atendida, ou seja, se prazos especificados para as transações estão sendo obedecidos e, se os limites de imprecisão definidos para os itens de dados não foram excedidos sendo realizado através da função de monitoramento.

\section{CONCLUSÃO}

A automação hospitalar é uma área multidisciplinar que envolve diversas áreas e por este motivo implica que estudos nesta área é muito abrangente e, portanto, envolvem uma vasta gama de conhecimentos, necessidades e restrições. Aumentando, a cada dia, a necessidade de transformar o grande volume de dados úteis e válidos em informações estratégicas e precisas em todas as áreas, mas precisamente nos ambientes hospitalares por tratar-se de vidas humanas.

Dessa forma, os sistemas computacionais estão em constante evolução e cada vez mais presentes em todas as áreas de conhecimento. Além disso, seus usuários estão cada vez mais exigentes quanto à qualidade de seus serviços, considerando tanto a consistência lógica quanto temporal, de maneira que garantam o desempenho do sistema.

Assim, com SGBD-TR é possível garantir as restrições de tempo impostas aos dados e transações, através de uma comunicação eficiente entre PS e o servidor do hospital, de forma que os mesmos possam acompanhar o monitoramento e sejam alertados de qualquer alteração nos sinais vitais do paciente em tempo hábil.

\section{REFERÊNCIAS BIBLIOGRÁFICAS}

1. Brooks, J.; Brooks, L. Automation in the medical field. Engineering in Medicine and Biology Magazine, IEEE Volume 17, Issue 4, July-Aug. 1998 Page(s):76, 81.

2. Murakami, Alexandre; Gutierrez, M. A.; Lage, Silvia Helena Gelas; Rebelo,Marina de Fátima de Sá; Ramires, José Antonio Franchini. A Continuous Glucose Monitoring. IEEE Computers in Cardiology, v. 32, p. 10-14, 2006. 
3. Leite CRM, Sizilio GRA, Neto ADD, Valentim RAM, Guerreiro AMG. A fuzzy model for processing and monitoring vital signs patients. BioMedical Engineering online, 2011.

4. Varshney, U. Patient monitoring using infrastructure - oriented wireless LANs. International Journal of Electronic Healthcare, Volume 2, Number 2 / 2006, 149163, 2006.

5. Neumann, P. G. Forum on Risks to the Public in Computers and Related Systems. The Risks Digest. Volume 25: Issue 21. ACM Committee on Computers and Public Policy, Peter G. Neumann, moderator. 2008. Disponível em: <http://catless.com/Risks/25.21.html>.

6. Baura, G.D. System theory in industrial patient monitoring: an overview Engineering in Medicine and Biology Society, 2004. IEMBS '04. 26th Annual International Conference of the IEEE. Volume 2, 2004 Page(s):5356 - 5359 Vol.7. 2004.

7. Pizarro, P. J. C.; 2003. MonitorIP . Monitoramento de Sinais Vitais através de uma Rede IP. Florianópolis. Dissertação (Mestrado em Engenharia Elétrica). Centro Tecnológico, Universidade Federal de Santa Catarina.

8. Araújo, G., B. Modelo Arquitetural de Comunicação para Monitoramento de Pacientes Baseado em Middleware, Computação Móvel e Ubíqua. Natal -RN. Dissertação Mestrado em Engenharia Elétrica e Computação. Universidade Federal do Rio Grande do Norte, 2010.

9. Carvalho, S., A., M. Um Sistema de Monitoramento Remoto de Pacientes usando Rede sem Fio. Dissertação de Mestrado da Pós- Graduação em Ciência da Computação da Universidade Federal de Minas Gerais, 2005.

10. Feng, David Dagan. Biomedical Information Technology(Series-Academic Press Series In Biomedical Engineering). ISBN:0123735831. Hardcover. Academic Press. 2007.

11. Marani, R., Perri, G.A. Design Of Advanced Electronic Biomedical Systems. International Journal of Advances in Engineering \& Technology, July 2012.

12. K. Ramamritham, S. Son, and L. DiPippo. Real-Time Databases and Data Services. Real-Time Systems, 28:179-215, 2004.

13. M. Amirijoo, J. Hansson, and S. H. Son. Specification and management of QoS in real-time databases supporting imprecise computations. IEEE Transactions on Computers, 55(3), 2006.

14. Shanker, U., Misra, M., and Anil K. Sarje, "Distributed Real Time Database Systems: Background and Literature Review" International Journal of Distributed and Parallel Databases, Springer Verlag, vol. 23, issue 2, pp 127-149 (April 2008). 
15. N. Idoudi, C. Duvallet, R. Bouaziz, B. Sadeg, and F. Gargouri. Structural model of real-time databases. In Proc. Of the 10th Intl. Conference on Enterprise Information Systems Databases and Informations Systems topic (ICEIS'08), volume 1, pages 171-178, Barcelona - Spain, 2008.

16. J. Hansson and S. H. Son. Overload management in rtdbs. In Real-Time Database Systems - Architecture and Techniques, pages 125-140. Kluwer Academic Publishers, 2001.

17. Jha, K. A., Xiong M., and Ramamritham, K. Mutual consistency in real-time databases. In RTSS, pages 335-343, 2006.

18. Lindstrom, J. Real time Database Systems. Solid, an IBM Company. Helsinki, Finland. March 25, 2008.

19. Lindstrom, J. Optimistic Concurrency Control Methods for Real-Time Database. Tese (Doutorado) — Department of Computer Science, University of Helsinki Finland, January 2003.

20. Wang, J., Han, S., Lam, K., Mok, K. A. Maintaining data temporal consistency in distributed real-time systems. Springer Science Business Media, LLC 2012.

21. N. Idoudi, C. Duvallet, R. Bouaziz, B. Sadeg. How to model a real-time database. UFR des Sciences et Techniques, 2009.

22. Squadrito, M. A. Extending the Priority Ceiling Protocol Using Read/Write Affected Sets. Dissertação (Mestrado) — Department of Computer Science, University of Rhode Island, 1996.

23. Brayner, A. Transaction Management in Multidatabase Systems. [S.1.]: Shaker Verlag, 1999. ISBN 3-8265-6142-2.

24. Ribeiro Neto, P. Mecanismos de Qualidade de Serviços para o Gerenciamento de Dados e Transações em Tempo-Real. Tese de Doutorado. Universidade Federal de Campina Grande, 2006.

25. Fernandes, Y.Y.M. P. Técnica de Controle de Concorrência Semântico para Sistemas de Gerenciamento de Bancos de Dados em Tempo-Real. Dissertação de Mestrado. Universidade Federal de Campina Grande, 2005. 